Revue des patrimoines

33 | 2017

Patrimoine culturel immatériel et institutions patrimoniales

\title{
De quelques prémices françaises du patrimoine culturel immatériel
}

Some early French premises of the intangible cultural heritage

\section{Philippe Mairot}

\section{(2) OpenEdition}

Journals

Édition électronique

URL : http://journals.openedition.org/insitu/15597

DOI : $10.4000 /$ insitu. 15597

ISSN : 1630-7305

Éditeur

Ministère de la culture

Référence électronique

Philippe Mairot, « De quelques prémices françaises du patrimoine culturel immatériel », In Situ [En

ligne], 33 | 2017, mis en ligne le 03 novembre 2017, consulté le 02 mai 2019. URL : http://

journals.openedition.org/insitu/15597; DOI : 10.4000/insitu.15597

Ce document a été généré automatiquement le 2 mai 2019.

\section{cc) (1)}

In Situ Revues des patrimoines est mis à disposition selon les termes de la licence Creative Commons Attribution - Pas d'Utilisation Commerciale - Pas de Modification 4.0 International. 


\title{
De quelques prémices françaises du patrimoine culturel immatériel
}

\author{
Some early French premises of the intangible cultural heritage
}

\author{
Philippe Mairot
}

1 L'élection de tel ou tel morceau du monde, animé ou inanimé, humain ou non-humain, comme patrimoine, doit être corrélée, non pas seulement à des qualités intrinsèques et substantielles propres, qui seraient à chercher dans cet objet et le feraient surgir spontanément, immédiatement, par lui-même, devant notre admiration et notre reconnaissance, mais à ses électeurs, dans un contexte donné précis, dans le conflit ou dans l'enchantement. Comment les experts de ces mêmes objets sont-ils embarqués dans des mouvements sociaux et symboliques débordant largement les enjeux scientifiques (mais que la science doit objectiver)? Comment embarquent-ils avec eux, ou non, les fameuses communautés? Comment, entre ces deux voies également exposées, naviguentils parmi les récifs de l'assignation ou de la ré-assignation identitaire, les chants des sirènes de l'Un indemne d'autrui, les courants de la marchandisation du patrimoine, les vents de l'expertise technocratique, isolée et élitiste? Cette attention aux procédures sociales de désignation des patrimoines n'est plus seulement ce lieu désormais commun de la vigilance scientifique. Elle a pris corps comme norme au cœur de la définition mondiale du patrimoine culturel immatériel. C'est en quoi la convention de l'Unesco de 2003, ratifiée par 174 États en mai 2017, dont la France en 2006, ne constitue pas seulement une étape nouvelle de l'extension du domaine patrimonial, en y incluant ce qui n'a pas de consistance matérielle. Comment les anciens et durables questionnements sur la nature du patrimoine - quel patrimoine ? qui le désigne ? qui en jouit ? - se réfractentils dans la lumière de cette nouvelle convention?

2 Nous voudrions tenter ici un rappel de quelques précédents et questionnements anciens français -, derrière l'apparente nouveauté de ce concept souvent présenté comme d'inspiration orientale, sous la forme d'un retour à Quatremère de Quincy, à Chanzeaux, aux écomusées, et d'un détour (amer) par Chebika. 
On pourrait, dans une première et vague approximation, considérer que c'est affaire de vocabulaire et que le folklore, la culture populaire, les arts et traditions populaires, aujourd'hui le patrimoine culturel immatériel, sont seulement les affections passagères et contingentes de la mode, dénommant autrement un domaine inchangé, stable, permanent et qui désigne, au fond, toujours les mêmes contenus, mis au jour par les mêmes procédures de production: à savoir, par exemple, l'investigation historique, l'enquête de terrain.

4 En effet, ce n'est pas le fait que le patrimoine embrasse les traces, y compris immatérielles, du peuple, des pauvres, des paysans, des ouvriers ou des dominés qui constitue une vraie révolution. Depuis le xvIII ${ }^{\mathrm{e}}$ siècle, cet intérêt érudit ou savant puis romantique pour le peuple et ses mœurs apparait dans nos sociétés et se manifeste dans des collectes de littérature orale et d'objets. L'Académie celtique n'inclut-elle pas parmi les monuments les traditions, croyances, coutumes, etc. ?

Quatremère de Quincy conteste en $1796^{1}$ les « rapatriements d'œuvres d'art ", évoquant cette

foule d'objets sans valeur pour l'étude, désormais sans rapport avec les idées qui leur donnaient la vie. [...] Ne nous dites plus que ces ouvrages de l'art se conservent dans ces dépôts. Oui, vous y avez transporté la matière; mais avez-vous pu transporter avec eux ce cortège de sensations tendres, profondes, mélancoliques, sublimes ou touchantes qui les environnait? Avez-vous pu transférer dans vos magasins cet ensemble d'idées et de rapports qui répandait un si vif intérêt sur les œuvres du ciseau ou du pinceau? Tous ces objets ont perdu leur effet en perdant leur motif. Le mérite du plus grand nombre tenait aux croyances qui leur avaient donné l'être, aux idées avec lesquelles ils étaient en rapport, aux accessoires qui les expliquaient, à la liaison des pensées, qui leur donnait de l'ensemble. [...] Que me disent toutes ces effigies qui n'ont plus conservé que leur matière? [...] Déplacer tous les monuments, en recueillir ainsi tous les fragments décomposés, en classer méthodiquement les débris, et faire d'une telle réunion un cours pratique de chronologie moderne ; c'est pour une nation vivante se constituer en état de nation morte ; c'est de son vivant assister à ses funérailles; c'est tuer l'art pour en faire l'histoire ; ce n'est point en faire l'histoire mais l'épitaphe.

Paul Valéry ${ }^{2}$, un peu plus d'un siècle plus tard, ne dit pas autre chose, et guère autrement, dans son fameux texte sur « le problème des musées ", évoquant le " voisinage de visions mortes » et l'errance d'objets privés de leur lumière, de leur espace et de leurs alliances. (On croirait lire précisément la description de la boutique d'antiquités dans La Peau de chagrin.)

7 Que le patrimoine, pour être saisi intelligemment et complètement, doive inclure, autour des œuvres, et aussi bien, des objets, outils, espaces, bâtiments, paysages, - toutes catégories très distinctes les unes des autres mais qui ont en commun d'être matérielles les représentations, croyances, savoirs, savoir-faire, usages, rituels, pratiques, etc. toutes catégories non moins distinctes les unes des autres mais qui ont en commun d'être immatérielles - qui leur sont associés dans la pratique, ce n'est donc une nouveauté ni pour la recherche ni pour le monde du patrimoine.

8 Cette distinction entre «matériel » et «immatériel », qui a pu obnubiler quelques lecteurs de cette convention, n'est-elle pas d'ailleurs un peu rustique quand elle s'applique aux phénomènes humains? Comment, dans le monde humain, pourrait-on appréhender un élément seulement matériel, qui ne serait pas pensé? Et si, par hypothèse, cela pouvait se faire, comment pourrait-on lui reconnaitre une valeur 
patrimoniale, c'est-à-dire immatérielle, sans affecter son statut d'être "purement matériel »? Nous ne pourrions saisir, quelque effort d'abstraction que nous mettions en œuvre, que des objets déjà humanisés, réfléchis, autrement dit affectés de valeur: augmentés d'immatérialité.

Pour l'ethnologie, c'est évidemment tout un: c'est cet ensemble dynamique qui est pertinent, formé par l'intrication sans cesse à refaire entre les choses et les savoirs, les représentations, les mémoires, les valeurs, les croyances faites choses, au sein de redéfinitions continues des domaines respectifs de la matière et de l'esprit, de l'humain et du non-humain. Du reste, prudemment et justement, l'article 2 de la convention le précise : «[...] ainsi que les instruments, objets, artefacts et espaces culturels qui leur sont associés » (fig. 1).

Figure 1

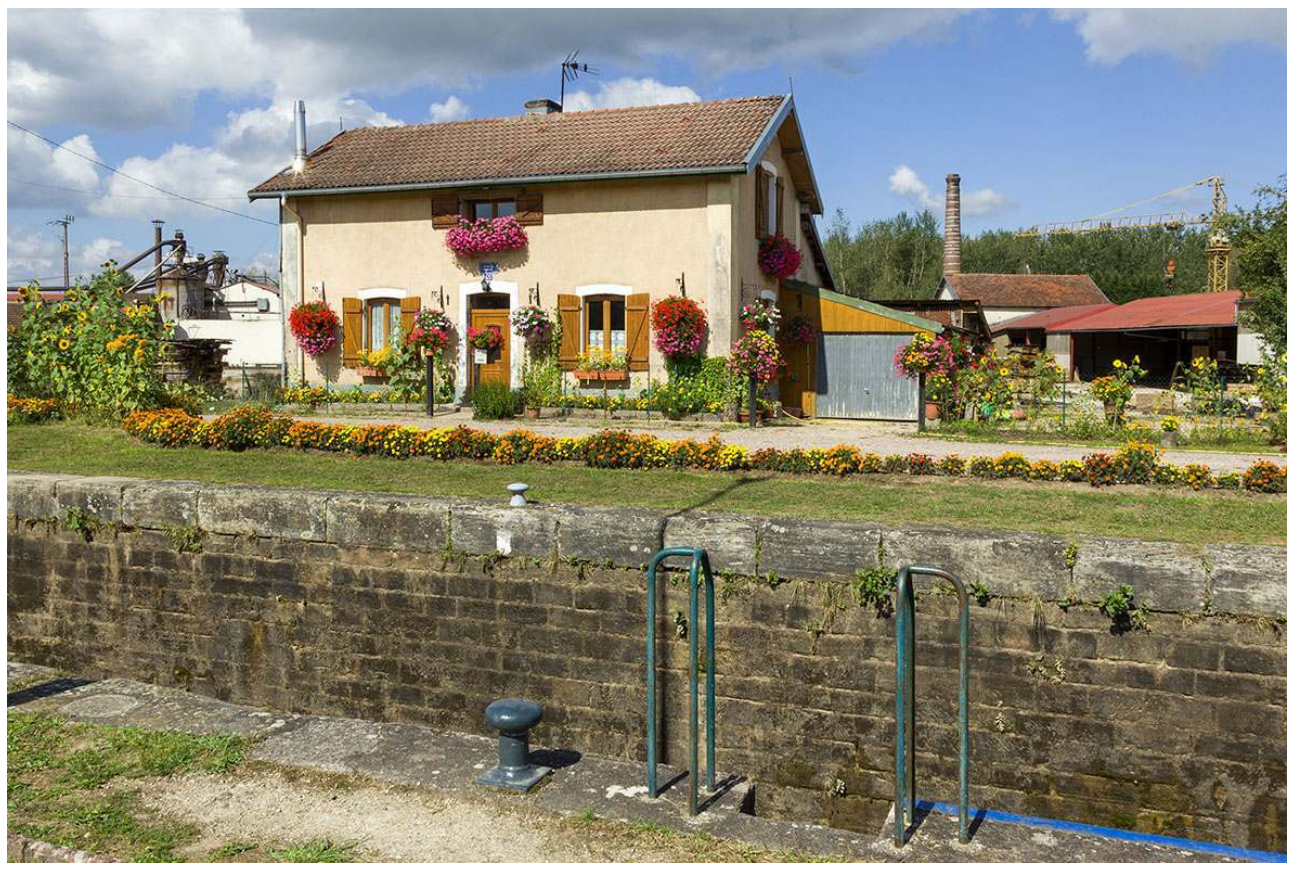

L'écluse fleurie de Corre (Haute-Saône).

Phot. Sonia Dourlot, 2015. @ Région Bourgogne-Franche-Comté, service Inventaire et Patrimoine.

10 S'il est tentant de prétendre que le patrimoine doit désormais inclure sa matière noire (invisible mais déduite, indispensable hypothèse pour rendre compte du visible, de par la masse manquante de l'univers), la comparaison s'arrête là car la physique n'attend pas de la matière noire qu'elle prenne conscience d'elle-même pour valider son existence. Tandis que la définition du patrimoine culturel immatériel l'inclut: l'être en soi ne lui suffit pas, la conscience de soi est une condition sine qua non de son existence.

11 Concentrons-nous donc plutôt sur cette distinction trop souvent restée dans l'ombre mais combien décisive de lieu de formation de cette conscience, de ses liens avec les groupes humains dans leurs reconfigurations et re-liaisons. Accordant apparemment plus de place au lien qu'au bien, ce texte fait-il surgir un ou plusieurs acteurs nouveaux dans la production du patrimoine? Déqualifie-t-il l'expert? (Son latin?) Mais si l'autre savait mieux? 
12 En caricaturant à gros traits l'épistémologie des sciences de l'homme, on peut distinguer sur ce plan deux voies d'abord: celle qui s'inscrit dans la perspective où la science est reine parce que le sens est caché; le sens de leurs actions est celé aux acteurs mêmes. Trompés, ils sont joués ou aveugles et la "science» seule, de par sa procédure d'objectivation (rupture épistémologique avec les sensations premières et fallacieuses apparences) dénonce le leurre et fait apparaître en pleine lumière le caché : la nuit recule. Historiquement, l'ethnologie affirme la possibilité de constitution d'un type de savoir sui generis portant sur les cultures humaines, par un étranger, au terme d'une pratique d'immersion dans une culture autre que la sienne, type de savoir spécifique qui diffère de celui que ces groupes humains se font sur eux-mêmes. Entre les deux, un écart : d'une part, le savoir qu'on dit scientifique pour indiquer qu'il cherche à respecter un ensemble de procédures et qu'il soumet ses énoncés de son propre chef au jugement de la communauté : la seule propre à - momentanément - attester la valeur de ses assertions : la communauté scientifique (déterritorialisée) et, d'autre part, ce qu'un groupe connaît de son être de groupe : par exemple ses limites, temporelles et spatiales, son origine, ses rapports avec les autres, la manière dont il pense perdurer au fil du temps, dont il organise ses divisions internes, ses hiérarchies, ses croyances, ou encore la place et la valeur qu'il accorde aux bêtes, végétaux, esprits, morts, bactéries, récits, etc.

Cet écart est-il d'une autre nature que celui qui oppose au sein d'un seul et même groupe humain, à un moment donné, les propositions jugées vraies qui pourraient instituer un monde commun à transmettre et celles qui sont jugées fausses, discréditées? Une abondante littérature politique et épistémologique lui a été consacrée, souvent à partir d'un extensible ensemble de couples d'opposition: idéologie versus science, croyance versus savoir, illusion versus vérité, apparence sensible versus sens caché. Derrière ces concepts, les hommes : ceux qui ignorent leurs conditions réelles d'existence et ceux qui les connaissent; ceux qui sont dans la nuit, privés de cette capacité d'être des sujets du savoir de soi et condamnés à être objectivés par d'autres : ceux qui les éclairent. Règne alors le vocabulaire du dévoilement, du décryptage, de la démystification, du dessillement.

Le musée, ou la politique patrimoniale qui découle de cette posture, est le musée classique, où la civilisation qui étudie les autres et les désigne réunit à sa guise les objets qu'elle considère, quant à elle, comme significatifs et porteurs de sens et de valeurs, souverainement en dévoile la vérité. Et, les sommant, ces objets, elle somme les individus de s'y identifier. L'esthète selon Sartre est celui qui étudie les hommes comme si c'étaient des fourmis ${ }^{3}$.

15 Soit, seconde voie de notre caricaturale distinction, rupture avec la rupture, qui admet que les acteurs jouent en toute conscience, savent ce qu'ils font, sont pleinement producteurs du sens de leurs actions, et l'on parle alors plutôt, à leur propos, de stratégie: la «science humaine » n'a aucun privilège d'extériorité, joue de plain-pied dans le même monde, acteur parmi les autres, contestable et indéfiniment métabolisable. Ce que pensent les particules de notre manière de parler d'elles, nous ne le savons pas, mais les spécialistes, depuis le début $\mathrm{du} \mathrm{xx}^{\mathrm{e}}$ siècle, disent que les observations des observateurs, à une certaine échelle, affectent les phénomènes et que nous observons de facto aussi notre propre présence agissante. Ce que pensent les hommes du Paléolithique de ce que nous racontons sur le sens des peintures que nous leur attribuons, nous ne le savons pas non plus. Une des spécificités de l'ethnologie est de risquer ses énoncés relatifs à un groupe humain, non seulement dans les instances de critique et validation 
des savoirs mais au sein même des groupes objets: la "restitution» a été théorisée comme un moment de la recherche (et les gens de ma génération ont été formés ainsi), un moment d'ailleurs problématique selon Françoise Zonabend qui évoque ce « décepteur du social ${ }^{4} »$.

Exemple lumineux et savoureux pour notre contexte: l'expérience des habitants de Chanzeaux, village d'Anjou, qui réfutent partiellement l'image, selon eux passéiste et nostalgique, produite par les ethnologues dirigés par Laurence Wylie, venu de Harvard, qui les ont étudiés entre 1957 et $1965^{5}$. Ceux-là sont gentiment accusés de définir des images idéales pour l'éternité et ne pas accepter le changement permanent qui est la vie, de vouloir les enfermer dans une vision fatale et archaïque de leur passé, idéalisé, qui n'était pour eux qu'un moment de leur histoire, quand l'ethnologue voulait en faire, selon eux, une nature quasi éternelle, à laquelle ils auraient dû se conformer indéfiniment pour rester les mêmes. Critiques qu'ils synthétisent dans cette formule goûteuse : "Vous êtes plus conservateurs que nous ». On songe à la formule (attribuée à Gustav Mahler, inspirée possiblement du sonnet 73 de Shakespeare, puis de John Denham) : « La tradition n'est pas la conservation des cendres mais l'entretien du feu. »

Voici l'expert objectivé, ramené à ses présupposés. Le peuple sort de l'éprouvette, n'est plus objet, revendique un statut de sujet de ses représentations comme de son histoire. Les voilà qui "répondent », comme on le dit d'enfants insolents qui osent s'opposer à la parole d'autorité parentale, ou de ceux qui protestent lors d'un contrôle d'identité, et refusent d'être enfermés, réifiés par d'autres, assujettis dans un folklore.

Le musée, ou la forme patrimoniale qui découle de cette posture, qui prend au sérieux la voix des sans-voix, s'incarne en France à partir de 1971 dans les écomusées, et ailleurs dans le monde, dans des expériences qui se nomment musées de voisinage ou musées communautaires. C'est dans les Lettres à Miranda ${ }^{6}$ que Quatremère de Quincy a cette formule: «C'est avec vérité qu'on peut dire que le pays fait partie du muséum de Rome. Que dis-je ? Le pays est lui-même le muséum 7 .» (fig. 2) 


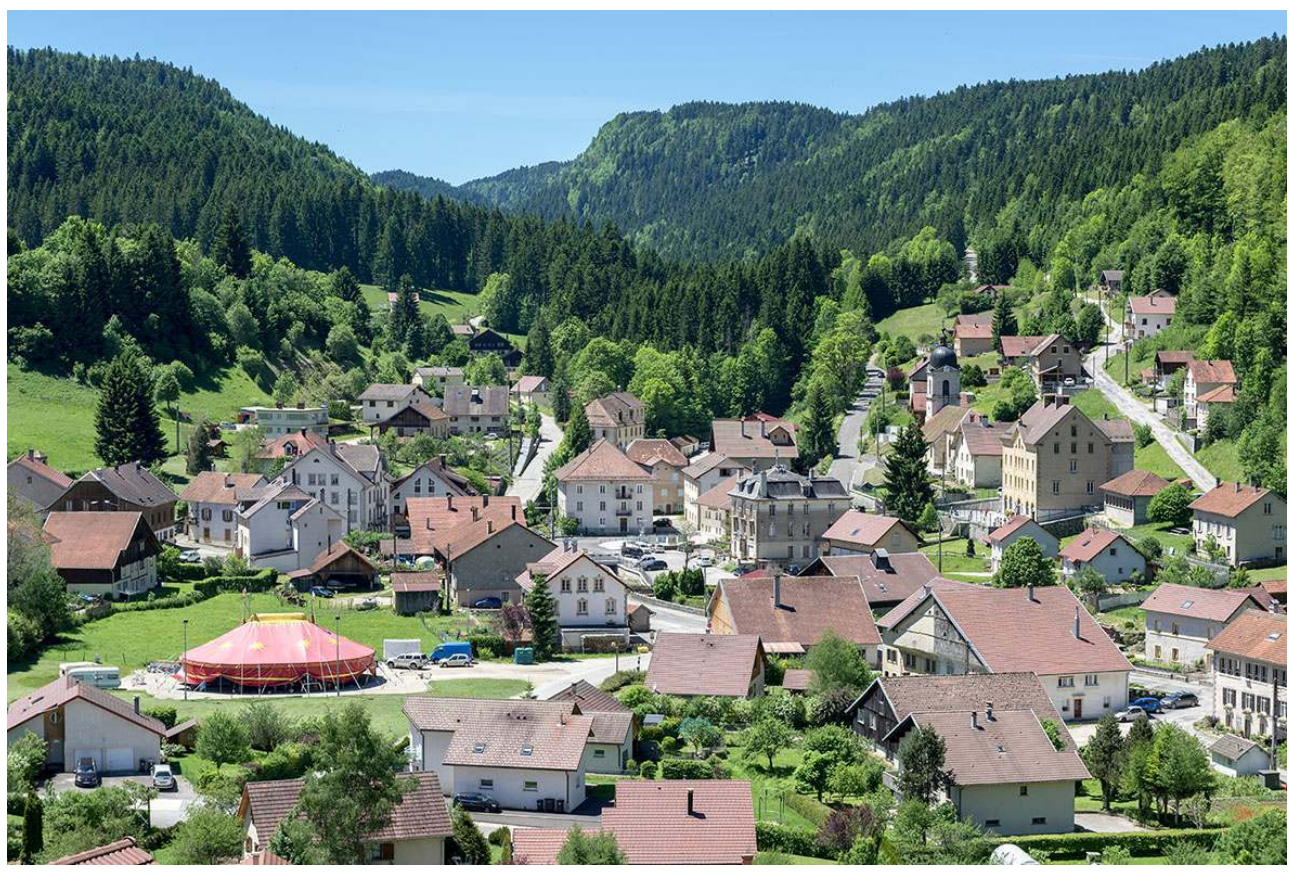

« Le pays est lui-même le museum »: Les Gras (Doubs).

Phot. Sonia Dourlot, 2017. @ Région Bourgogne-Franche-Comté, service Inventaire et Patrimoine.

Un siècle et demi plus tard, la même obsession peut se lire au travers de la naissance des écomusées. Après l'apparition des politiques d'aménagement du territoire (création de la Datar en 1963), après celles des parcs naturels régionaux (1967), du musée de plein air de Marquèze (Landes) en 1969, préoccupations environnementales, pluridisciplinarité, conservation in situ, extension du domaine patrimonial, sont peu à peu combinées, sous l'impulsion de Georges Henri Rivière, dans des nouveaux établissements patrimoniaux, dits « écomusées » en 1971. La même année, l'Icom (International Council of Museums) inclut la question du développement dans les missions du musée. La notion de musée « intégral » est évoquée lors de la table ronde de Santiago (Chili) en 1972. Avec Hugues de Varine et l'écomusée du Creusot, (musée de l'Homme et de l'Industrie) à partir de 1971-1972, l'enjeu scientifique de la prise en compte du contexte et du monde industriel se double d'une exigence qu'on pourrait qualifier d'éthique et politique, incluant les habitants dans ce fameux contexte. Dès lors, le patrimoine consiste indissociablement en bien et lien social, processus d'engendrement, - d'auto-engendrement.

20 Ce pays est habité et les populations n'y sont pas des objets mais doivent être au contraire parties prenantes du projet : des sujets, au sens de la tentation moderne par excellence, celle de l'auto-institution, de l'auto-fondation. C'est pourquoi les habitants siègent dans les instances de décision de l'écomusée au sein du comité des usagers. Hugues de Varine formule ainsi en 1973 cette radicalité où nous pouvons entendre comme l'écho de Quatremère de Quincy : "La communauté tout entière constitue un musée vivant dont le public se trouve en permanence à l'intérieur. Le musée n'a pas de visiteurs, il a des habitants ${ }^{8} . »$

21 Cette identité du musée et du pays touche évidemment à l'aporie: si le monde est le musée, où est le musée ? Il n'y a plus de musée. Comment le geste patrimonial, qui se caractérise par le désir de mettre à part (de traiter sélectivement, élire, discerner du 
commun certains objets, selon un certain nombre de critères), pourrait-il simultanément intégrer l'idée de tout inclure - parfois caricaturée sous le terme de: «tout patrimoine »?

Mais Georges Henri Rivière et Hugues de Varine sauvent la définition du néant en l'ancrant dans la population, qui est sa vraie et seule légitimité. C'est bien alors une posture neuve que d'exiger que le musée ou la politique patrimoniale soient non seulement au service des populations mais que celles-ci en soient le cœur, la raison d'être. On passe d'une acception d'un patrimoine désigné (validé, authentifié) par les experts extérieurs soumis à l'examen - toujours provisoire - de la communauté scientifique, supposant des populations résignées au contrôle d'identité, à une autre où les habitants sont au centre?.

Georges-Henri Rivière écrit en 1976, très conscient des dangers et ambivalences : « Il y a, de la part des responsables, le risque de mettre une population en cage à la manière d'un animal au zoo, et le risque de manipuler cette population. Il y a les équivoques d'un statut flottant entre autogestion et tutell ${ }^{10}$.» (fig. 3) Certes, l'écomusée embrasse un patrimoine étendu aux témoignages (qu'on ne disait pas matériels) des mondes ruraux et industriels (comme les outils ou les costumes) ainsi qu'aux mémoires et pratiques sociales, représentations (qu'on ne qualifiait pas d'immatérielles) mais surtout, il en éprouve la valeur au travers d'une nouvelle épreuve: il en soumet la validité aux populations elles-mêmes, contraignant plus ou moins la science et ses critères à s'exposer au monde non savant dans des délibérations ouvertes et jamais closes, à mesurer comment ce savoir affecte les groupes humains.

Figure 3

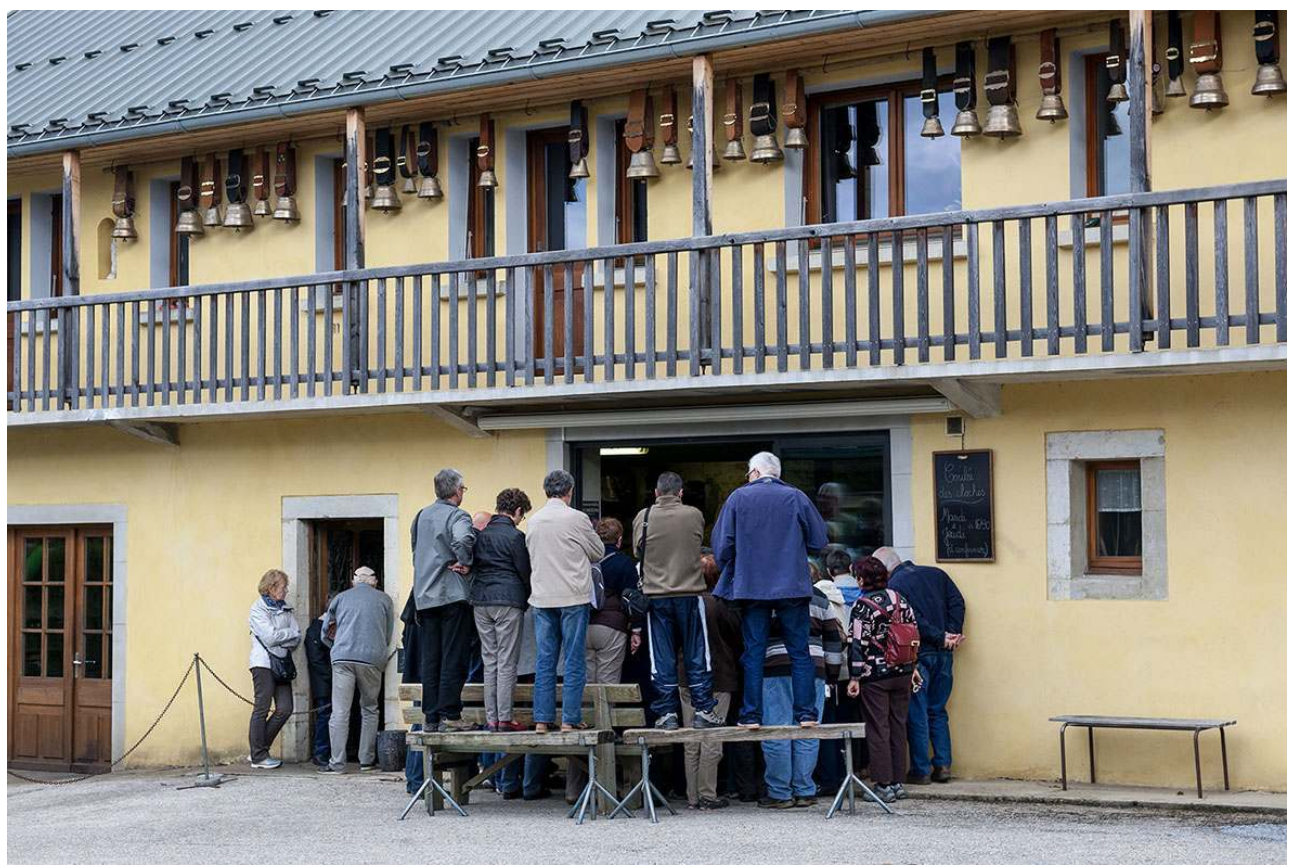

Visiteurs devant la fonderie de cloches Obertino à Labergement Sainte Marie le 24 septembre 2015 (Doubs).

Phot. Jérôme Mongreville, 2015. (C) Région Bourgogne-Franche-Comté, service Inventaire et Patrimoine. 


\section{(fig. 4) ?}

Figure 4

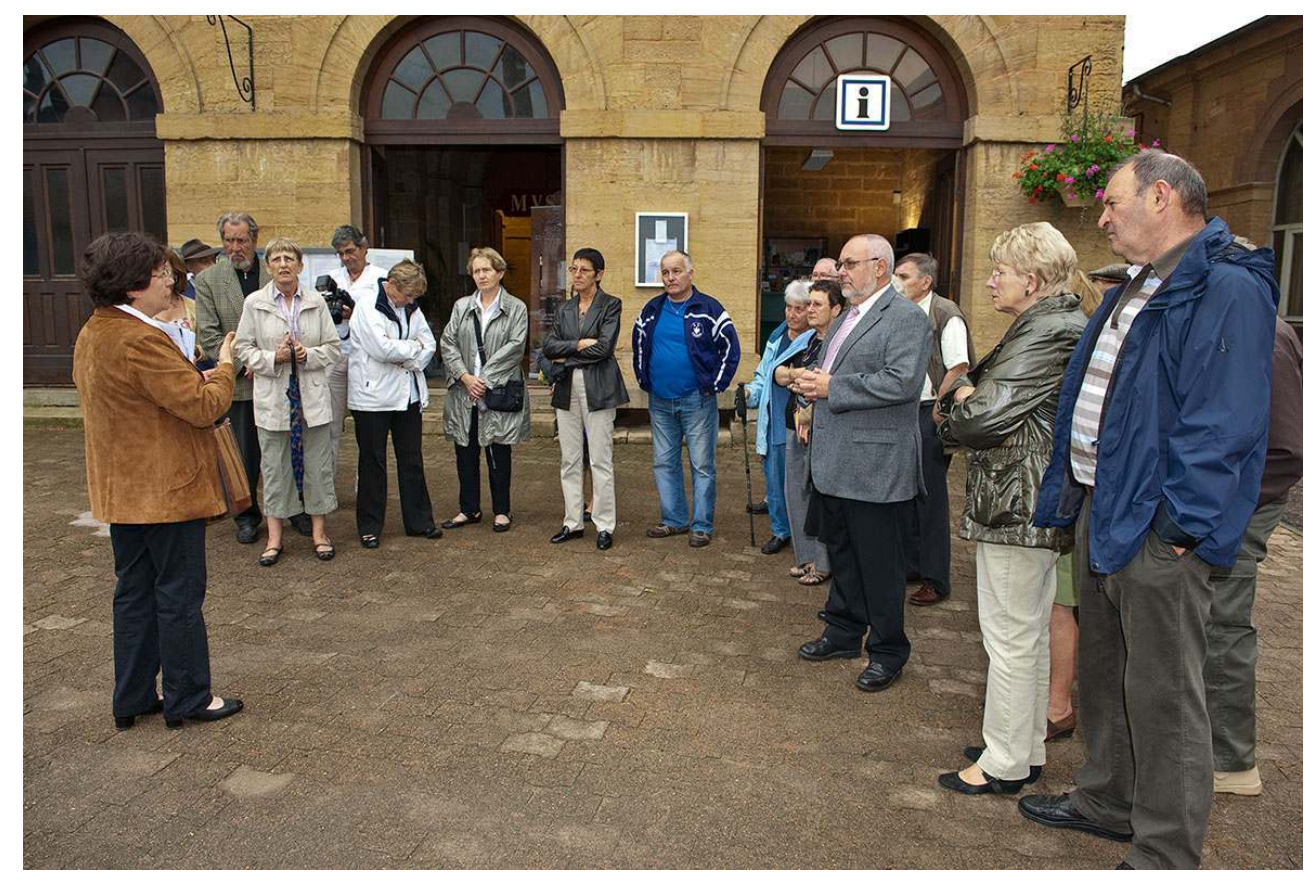

L'expert et les habitants : conférence lors des journées du patrimoine à Rougemont en 2011 (Doubs). Phot. Jérôme Mongreville, 2011. (C) Région Bourgogne-Franche-Comté, service Inventaire et Patrimoine.

Dans ce modèle écomuséal, la place de l'expert n'est cependant pas celle du siège vide ; elle voudrait s'apparenter plutôt à celle du philosophe dont Socrate nous a fourni le modèle : non plus celui qui sait et dont le savoir peut couler, d'un cerveau savant à un autre cerveau ouvert. Mais celui qui sait qu'il ne sait pas et qui est plus préoccupé par l'autre que par lui-même et son propre savoir: son projet est de faire progresser son interlocuteur, dans le lien vivant du dialogue, à la recherche du beau et du bien. Plutôt créer un sujet qui pense que transmettre un hypothétique savoir. Dans cette définition, les experts sont au service de la communauté et lui apportent matériaux et outils critiques pour la connaissance de l'histoire ainsi que des questionnements organisés sur les groupes, et les manières dont les forces et conflits les travaillent. C'est leur pleine responsabilité - non négociable - et cette connaissance, ils la risquent, l'offrent en partage, n'imposent plus mais s'exposent. Une certaine égalité de valeur semble prévaloir, où les différences de points de vue peuvent se confronter, les controverses se déployer, jouer. Critiquant l'idée selon laquelle la société pourrait s'autodéterminer, " comme si elle pouvait détenir un soi », le philosophe Peter Sloterdijk ${ }^{11}$ qualifierait plutôt l'animateur territorial de gourou de la communauté - coach - maitre du devenir 
communautaire au moyen de techniques de sortie des passivités et des inerties : l'histoire, les habitudes, la tradition qui chosifient ${ }^{12}$.

Cette ambition se diffuse bien au-delà des écomusées durant les années qui suivent. Elle est mentionnée dans le rapport Benzaï $\mathrm{d}^{13}$ en 1979, et André Chastel et Jean-Pierre Babelon évoquent, parmi les critères du patrimoine, "l'attachement » des indigènes ${ }^{14}$. Au niveau international, en 1994, le «document de Nara sur l'authenticité ${ }^{15}$ » reconnaît que "Le respect dû à la diversité des cultures exige que chaque œuvre soit évaluée avec des critères provenant du contexte culturel auquel elle appartient» et depuis 2003, la convention sur le patrimoine culturel immatériel «s'efforce d'assurer la plus large participation possible des communautés... » (art. 15) et définit ainsi le patrimoine culturel immatériel: «les pratiques [...] que les communautés, groupes et, le cas échéant, les individus, reconnaissent comme faisant partie de leur patrimoine culturel» (art. 2). Une telle formule échappe-t-elle à la tautologie ? Au solipsisme ? À la fortune d'une prédiction autoréalisatrice dont une des caractéristiques est donc d'être protégée des savoirs experts, du moins experts authentifiants comme avant, c'est-à-dire agents prétendument exclusifs de l'authentification? Est-ce à dire que le patrimoine culturel immatériel soit, par excellence, le sujet auquel on ne peut avoir accès en tant qu'expert, à moins de s'exprimer au nom de son propre groupe, et d'être reconnu comme tel ? Ce statut était naguère désigné-dénigré sous le terme d'érudit « local».

La convention (art. 2.3) reconnaît certes bien à la recherche une place dans la sauvegarde : là commence évidemment une autre série de questionnements relatifs à la manière dont s'organisent les controverses et avec qui : Les natifs? Les immigrés ? Quelles instances représentatives de quoi, ou qui, dans quel espace public, et dont la représentativité est validée par qui?

29 Si cette convention sanctionne la position des groupes locaux détenteurs de pouvoir, non celle des experts, que vient faire le "savoir scientifique », sa prudence procédurale, sa lente critique, dans ce champ social? Est-ce le pari de l'obscurantisme? Le retour en gloire des croyances, de l'idéologie, de la célébration de soi, de l'hagiographie, la rupture avec la rupture qui donnait à la science son rêve d'externalité objectivant?

Nous avons évoqué Chanzeaux. Nous pourrions aussi évoquer le dysphorique exemple de Jean Duvignaud revenant sur son terrain de Chebika, tel qu'il l'évoque dans Le Langage $p e r d u^{16}$. Les habitants qui, dans les années de l'enquête consécutive à la décolonisation, étaient les acteurs d'un travail de la communauté sur elle-même, centré sur des préoccupations de maîtrise d'un développement réfléchi et décidé, se trouvent réduits à l'état de figurants d'une prétendue identité, « minables montreurs de serpents » pour des touristes venus là précisément attirés par l'enquête ethnologique et sa promesse d'authenticité dévoilée. Dévoiement de la recherche, déchéance d'une population acculée à la singerie de "soi ", dégénérant en objet exotique. Lorsque Jean Duvignaud évoque le sort malheureux des habitants de Chebika, devenus attraction et objets exotiques dans l'économie touristique, les mots sont durs: Si Tijani, esquissant « une sorte de danse et tirant des vipères édentées pour effrayer et fasciner à la fois les touristes ", " n'est plus qu'un mannequin vidé de sa substance », le village «mime pour quelques pièces la vie qu'il redécouvrit au moment de l'intervention $»^{17}$.

31 La « valorisation touristique » y est présentée sous la forme d'un violent et insupportable déni du devenir, jugé d'autant plus ignoble qu'il ne trompe pas seulement les touristes ce dont on se moque? - mais les populations elles-mêmes. Serait-elle plus désirable, cette 
marchandisation, si les habitants la décidaient ? Il y aurait là une expérience de terrain à méditer pour désenchanter le patrimoine culturel immatériel.

On observe souvent, à juste titre, que l'infléchissement de cette définition témoigne d'un contexte mondialisé, où plusieurs définitions du patrimoine se conjoignent ou se combattent alors que la convention de 1972 manifestait encore clairement ses sources européennes. On discerne en revanche souvent une influence orientale à l'œuvre dans la notion de patrimoine immatériel : ce que nous pouvons donc maintenant nuancer, après avoir rappelé ces lointaines racines françaises, réinterprétées et synthétisées dans les écomusées tels que Rivière les a progressivement conceptualisés comme des laboratoires de la prise en compte simultanée des aspects immatériels des patrimoines et de l'implication des populations. Des laboratoires où s'expérimentait la critique des postures d'observateurs et d'observés, des relations entre les détenteurs symboliques du " patrimoine » et ses inventeurs, où ce rapport objet de science/sujet d'histoire pouvait se réfléchir. L'écomusée fut une des matrices de cette conception - à présent consensuelle qui fut d'abord une vive controverse, une machine de guerre destinée notamment à jeter du trouble dans les prétendues racines toutes faites, autrement dit faites par d'autres.

En outre, il y a un grand écart entre la conception du Trésor humain vivant, telle que par exemple la Corée l'a adoptée à partir de 1960, centrée sur un individu considéré comme porteur transitoire de valeurs et savoirs qui le transcendent (ou le nient) en tant que tel et font de lui un "spécimen ", et la notion au contraire fondamentalement collective de "trésor » telle qu'Hannah Arendt ${ }^{18}$ la théorise ou telle qu'elle apparaît dans cette convention de 2003 qui considère " éventuellement des individus » mais principalement des « groupes et des communautés ».

Si ni le groupe ni la communauté (caste, famille, fratrie, classe d'âge, corporation, parti, nation, genre, etc.) ne sont définis dans cette convention, c'est peut-être pour permettre l'hypothèse que ce "groupe " n'est pas toujours déjà là, ne précède nullement la définition du trésor, mais que celle-ci lui est coextensive : que c'est notamment dans ce geste continu qui lui fait désigner, nommer, saisir, inventer ce « bien-lien » qu'un groupe se constitue, s'invente, se transforme, s'apparaît à lui-même et aux autres, construit sans fin son commencement, car décidemment, le « commencemanque ».

Si l'authentique est dans le processus (de désignation) et non pas dans le bien, si le bien n'est que l'accessoire (contingent, transitoire) comment justifier et sur quelle valeur fonder sa sauvegarde (fig. 5) ? Cette convention se lit alors comme un compromis entre la reconnaissance théorique de la prééminence de la création sociale continue ("récréation », « enrichissement»), la recherche de "l'authenticité » qui voudrait se garder des processus patrimonialisants (id est figeant le vif, dénaturant, dégénérant en choses mortes) et une approche pragmatique soucieuse de "sauver des vestiges", d'afficher - comme une fatalité, malgré tout - l'idée de «sauvegarde patrimoniale ». C'est pourquoi une attention plus grande est accordée aux éléments jugés en danger évoqués dans l'article 13: "méthodologies de recherche pour une sauvegarde efficace du patrimoine culturel immatériel, en particulier du patrimoine culturel immatériel en danger. » C'est aussi l'objectif de la liste de sauvegarde (art. 16). 


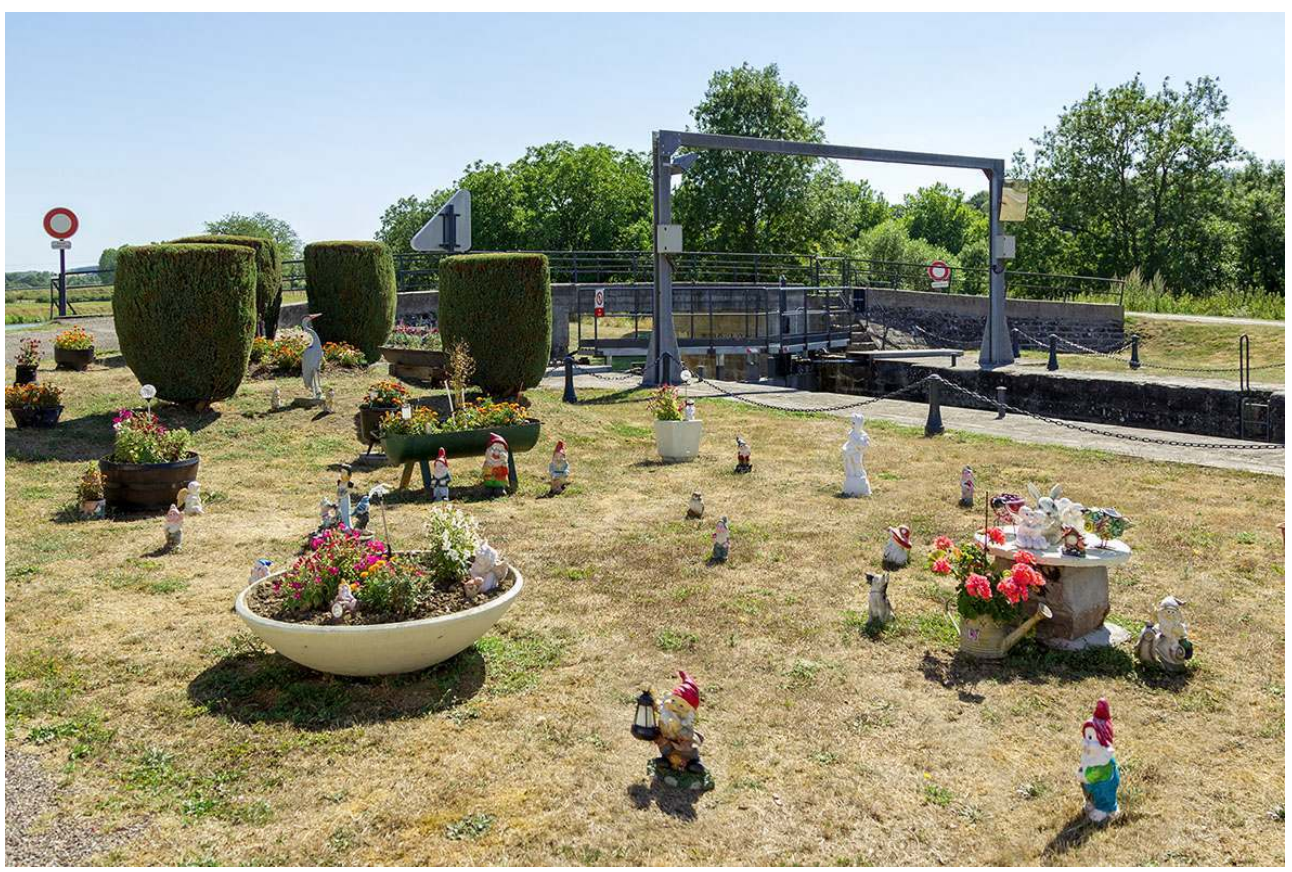

Composition décorative à l'écluse de Cendrecourt (Haute-Saône).

Phot. Sonia Dourlot, 2015. @ Région Bourgogne-Franche-Comté, service Inventaire et Patrimoine.

La menace qui pèse sur un phénomène évolutif est de ne plus évoluer, c'est-à-dire d'être patrimonialisé, comme l'a été par exemple le costume du pays d'Arles après que Mistral l'a muséifié. Cette notion de danger est essentiellement liée à une approche substantialiste de la culture, fondamentalement ethnostalgique ${ }^{19}$, qui redoute la déperdition des biens, l'éloignement d'avec la source pure d'authenticité, alors que la convention semble plutôt préférer une approche qui met l'accent sur la capacité des groupes à (se) créer sans cesse. Si une pratique est jugée (par qui ?) en danger, n'est-ce donc pas le signe d'un moindre attachement ou d'une moindre utilité ressentie par les praticiens, que l'esprit du texte considère, en dernier ressort, comme les meilleurs gardiens du processus patrimonial?

Pour conclure, nous pouvons nous demander si cela nous conduit à une critique ou à un abandon de l'ambition universaliste. Certes, nous observons qu'en apparence, 174 États (en mai 2017) se reconnaissent dans une seule et même définition du patrimoine. Mais dans sa substance, ce patrimoine est-il reconnu par tous, pour tous et pour chacun? Vaut-il pour tous, celui-là qui précisément doit sa valeur au fait qu'il est reconnu comme tel par un certain groupe ( communautés, groupes et, le cas échéant, des individus, qui créent, entretiennent et transmettent ce patrimoine", art. 15) à un certain moment de son histoire? Si c'est donc le groupe, dans les limites mouvantes de ses frontières (désignées par qui ?), qui est désormais le garant de l'authenticité du patrimoine culturel immatériel, c'est-à-dire du rapport vivant au patrimoine culturel immatériel, cela est aussi corrélé à un temps donné, autrement dit frappé d'une contingence fondatrice. Les groupes, entendus comme des entités dynamiques toujours se produisant elles-mêmes, pourraient tout aussi bien qualifier demain un tout autre patrimoine culturel immatériel ou n'en pas qualifier du tout, ou ne rien reconnaître de semblable, soit parce que ledit groupe aura cessé d'exister, soit encore parce qu'au sein dudit groupe, ce sont d'autres 
segments qui se seront saisis, ou non, de la question, d'autres locuteurs qui seront revêtus de l'autorité nécessaire et diront la valeur. C'est ainsi qu'Halbwachs abordait la question de la mémoire collective, en des termes fondateurs ${ }^{20}$. L'art. 2.1 de la convention de 2003 précise en effet que cela "procure un sentiment d'identité et de continuité », autrement dit, encourage à distinguer le sentiment d'identité ou de permanence de la réalité du changement continu.

De cela s'ensuit que ce patrimoine culturel immatériel, non plus défini et désigné à l'admiration de tous par des spécialistes étrangers, c'est-à-dire étrangers aux groupes humains concernés (détenteurs? héritiers? auteurs?), maintenant ancré dans les communautés vivantes - éligible universellement -, se trouve de facto immanent au local et au momentané, s'éloignant des valeurs d'universalité, de transcendance et d'éternité qui prévalaient naguère, du moins dans les idéaux, sinon dans les faits.

\section{NOTES}

1. - QUATREMÈRE de QUINCY, Antoine. Considérations morales sur la destination des ouvrages de l'art. Paris : 1815.

2. - VALÉRY, Paul. «Le problème des musées ». Dans CEuvres. Éd. Jean Hytier. Paris : Gallimard, coll. «Bibliothèque de la Pléiade », 1970, t. II. Pièces sur l'art [1931], p. 1290-1293.

3. - SARTRE, Jean-Paul. Critique de la raison dialectique. Paris : Gallimard, 1960, p. 183.

4. - ZONABEND, Françoise. «De l'objet et de sa restitution en anthropologie ». Gradhiva, 1994, ${ }^{\circ}$ 16 , p. 3-14.

5. - WYLIE, Laurence W. Chanzeaux, village d'Anjou [1966]. Paris : Gallimard, 1970.

6. - QUATREMÈRE de QUINCY, Antoine. Lettres sur l'enlèvement des ouvrages de l'art antiques à Athènes et à Rome, écrites les unes au célèbre Canova, les autres au général Miranda. Paris: A. Le Clère, 1836.

7. - QUATREMÈRE de QUINCY, Antoine. Lettres sur le préjudice qu'occasionneroient aux arts et à la science le déplacement des monumens de l'art de l'Italie, le démembrement de ses écoles et la spoliation de ses collections, galeries, musées, etc. par M. Quatremère de Quincy. Rome : 1815. En ligne : http:// www.purl.org/yoolib/inha/12774 [consulté le 26/05/2015].

8. - VARINE-BOHAN, Hugues de. "Un musée "éclaté" : le musée de l'Homme et de l'Industrie, Le Creusot-Montceau-les-Mines ». Museum, 1973, vol. 25, n 4, p. 242-249.

9. - MAIROT, Philippe. « Musées et société ». Ethnologie française, 1997, vol.27, p. 344-356 ; Id. "Identités et musées de société ». Dans LE GOFF, Jacques (dir.). Patrimoine et passions identitaires. Paris : Fayard/Éd. du patrimoine, coll. « Entretiens du patrimoine », 1998.

10. - RIVIÈRE, Georges Henri. La Muséologie selon Georges Henri Rivière. Paris : Dunod, 1989.

11. - SLOTERDIJK, Peter. Tu dois changer ta vie. De l'anthropotechnique. Paris : Libella-Maren Sell, 2011.

12. - MAIROT, P. « Aux marches du musée ». Dans FOURÈS, Angèle, GRISOT, Delphine et LOCHOT, Serge (éd.). Le Rôle social du musée: Agir ensemble et créer des solidarités. Charny: OCIM, 2011, p. 39-47.

13. - BENZAÏD, Redjem. Rapport sur l'ethnologie de la France: besoins et projets. Paris : la Documentation française, 1979. 
14. - CHASTEL, André, BABELON, Jean-Pierre. La Notion de patrimoine [1980]. Paris : Liana Levi, 1994.

15. - Unesco/Icomos. Conférence de Nara sur l'authenticité. Nara, 1-6 novembre 1994. Voir le site : http://www.icomos.org/fr/notre-reseau/comites-scientifiques-internationaux/liste-descomites-scientifiques-internationaux/179-articles-en-francais/ressources/charters-andstandards/186-document-de-nara-sur-lauthenticite [consulté le 13/10/2017].

16. - DUVIGNAUD, Jean. Le Langage perdu. Essai sur la différence anthropologique. Paris : PUF, 1973, passim et p. 20.

17. - Ibid, p. 20.

18. - ARENDT, Hannah. La Crise de la culture. Huit exercices de pensée politique. Paris : Gallimard, coll. « Idées », 1972.

19. - MAIROT, Philippe. «La valorisation du patrimoine industriel ». Dans Écomusées et musées de société, POUR, 1997, n 153, p. 63-71.

20. - HALBWACHS, Maurice. La Mémoire collective [1925]. Paris : PUF, 1950.

\section{RÉSUMÉS}

La convention de 2003 sur le patrimoine culturel immatériel est souvent interprétée comme une rupture radicale avec les définitions antérieures : occidentales, centrées sur la matérialité et le savoir d'experts. En y intégrant les dimensions immatérielles et en plaçant les communautés locales au cœur de la définition même du champ patrimonial, ce texte opérerait un renversement important. Nous cherchons ici, au contraire, à relier cette convention à une préoccupation aussi ancienne que la définition du patrimoine français, qui fait dépendre la qualité du témoignage matériel de la richesse des données contextuelles, lutte en faveur de la conservation in situ plutôt que de déplacements, assimilés à des destructions, et in fine, aboutit à l'inclusion - problématique - de la communauté dans le processus de conservation. Dans les Lettres à Miranda, où Quatremère de Quincy conteste en 1796 les « rapatriements d'œuvres d'art » et évoque cette «foule d'objets sans valeur pour l'étude, désormais sans rapport avec les idées qui leur donnaient la vie ", nous discernons des sources pour une archéologie du patrimoine culturel immatériel et des ferments de développements de la notion de contexte. On trouve une résurgence de cette prééminence accordée au contexte - incluant la communauté en position de sujet de son développement dans le modèle français des écomusées. On observe - en évoquant Chanzeaux et Laurence Wylie, ou Chebika et Jean Duvignaud - la méthode même d'une certaine ethnologie qui soumet ses énoncés non seulement à l'examen critique de la communauté scientifique, mais les partage - non sans risque - avec la communauté humaine qui est l'objet de sa recherche. La science et ses critères s'exposent ainsi au monde non savant, dans des délibérations ouvertes, et s'obligent à apprécier comment ce savoir affecte ou non les groupes humains et à privilégier le processus même d'invention du patrimoine en ce qu'il mobilise les acteurs comme un tout plutôt que la seule chose désignée. Que deviennent les notions d'expertise, d'authenticité et d'universalité si désormais le patrimoine consiste dans cet engendrement communautaire de ce qui, selon les termes de la convention, « procure un sentiment d'identité et de continuité »?

The 2003 intangible cultural heritage convention is often interpreted as a radical break with earlier definitions of heritage which were Western, centered on materiality and on the knowledge of experts. By incorporating intangible dimensions and by placing communities at the 
heart of the definition of what heritage is, this text appears to operate a major reversal. In this article, however, we try to understand this UNESCO convention in the context of preoccupations that are in fact as old as the definition of heritage in France, whereby the quality of material evidence is linked to the wealth of contextual data, which struggled for conservation in situ rather than displacement, seen to be the equivalent of destruction. This approach ultimately results in the inclusion of the community in the conservation process, although this inclusion raises its own problems. In his 'Letters in Miranda', in 1796, Quatremère de Quincy contests the 'repatriation of artworks' and refers to this 'crowd of objects without value for study, now unrelated to the ideas that gave them life'. Here perhaps are some sources for an archaeology of the intangible cultural heritage and seeds for the future development of the notion of context. This new attention given to the preeminence of the context, including the role of the community as a subject of its development, is to be found in the French model of eco-museums. Authors such as Chanzeaux, Laurence Wylie, or Chebika and Jean Duvignaud submit their statements not only to the scrutiny of the scientific community, but also share their observations with the human community which is the object of these observations. Science and its criteria thus expose themselves to the non-scholarly world, in open discussions, and undertake to assess how this knowledge affects human groups and to favour the process of invention of the heritage as it mobilises actors as a whole, rather than only the thing designated. What becomes the notions of expertise, authenticity and universality if heritage now consists of what a community generates and which, under the terms of the convention, 'provides a sense of identity and continuity'?

\section{INDEX}

Mots-clés : écomusée, patrimoine culturel immatériel, identité, communauté, expert

Keywords : eco-museum, intangible heritage, community, identity

\section{AUTEUR}

\section{PHILIPPE MAIROT}

Conservateur en chef du patrimoine, service Inventaire et Patrimoine, Direction culture, sport, jeunesse et vie associative, Région Bourgogne-Franche-Comté philippe.mairot@bourgognefranche-comte.fr 